\title{
A Content Addressable Memory for use in CEBAF's CLAS Detector Level 2 Triggering System
}

\author{
R.F. Hodson, D.C. Doughty Jr., D.C. Allgood, S.A. Campbell \\ Christopher Newport University, Newport News, VA 23606 \\ W.C. Wilson \\ NASA Langley Research Center, Hampton VA 23681 \\ M.H. Bickley \\ CEBAF, Newport News, VA 23606
}

\section{Abstract}

A collaboration of researchers from CEBAF, CNU and NASA is designing a $256 \times 32$ specialized Content Addressable Memory (CAM) for the level 2 triggering system in CEBAF's CLAS detector. These integrated circuits will find tracks and the momentum and angle of each track within 2 microseconds of an event. The custom CAM can operate as conventional memory, performing read and write operations, and can additionally perform independent byte compare operations across all words simultaneously. It is this compare feature which makes these CAMs attractive for identifying tracks passing through drift chambers by linking together segment number triplets within the CAM. Simulations have indicated that less than $16 \mathrm{k}$ triplets need to be stored for each sector of the detector. This implies the level 2 triggering can be performed with 64 CAM chips per sector, or 384 total. Each data channel into a sector CAM array is buffered in a FIFO and is designed to handle aggregate data rates up to $750 \mathrm{Mbs}$ for three channels (one channel/superlayer). The architecture of the level 2 trigger and details of the CAM chip design are discussed along with a performance report on our prototype CAMs.

\section{INTRODUCTION}

In CEBAF's large acceptance spectrometer (CLAS), the level 2 triggering system determines whether or not to digitize data associated with a detector event based on particle momentum and polar angle. This process is allotted $2 \mathrm{uS}$ per event in the triggering systems' time budget. Because of the demanding time constraints for this system, a hardware approach to track finding is being implemented based on a Content Addressable Memory (CAM) array. This paper focuses on the architecture and design of a custom CAM chip being developed at Christopher Newport University. This CAM-based level 2 trigger is an extension of the VLSI track finding research of Dell' orso and Ristori $[1,2]$.

\section{THE CAM IN LEVEL 2}

Particle location information (hit cells) from drift chamber superlayers are converted to segment addresses by a Xilinx Gate Array based Segment Finder. These segment addresses, which represent the position of a particle in a superlayer, are sent over three fiber channel data links to a CAM-based Track Finder. The function of the Track Finder is to link together triplets of segment address from each of the superlayers and determine if this triplet corresponds to a realizable particle trajectory. A realizable segment triplet is called a road. Identified roads are then sent to a Kinematic Lookup Table which determines the particle's momentum (p) and polar angle $(\theta)$. The momentum and polar angle can be compared against a range of angles and momenta in which experimenters are interested. If the event data are within the experimenter's specified ranges, a level 2 trigger occurs initiating data conversion and readout from all six sectors of the CLAS detector. Figure 1 shows a block diagram of the level 2 triggering system.

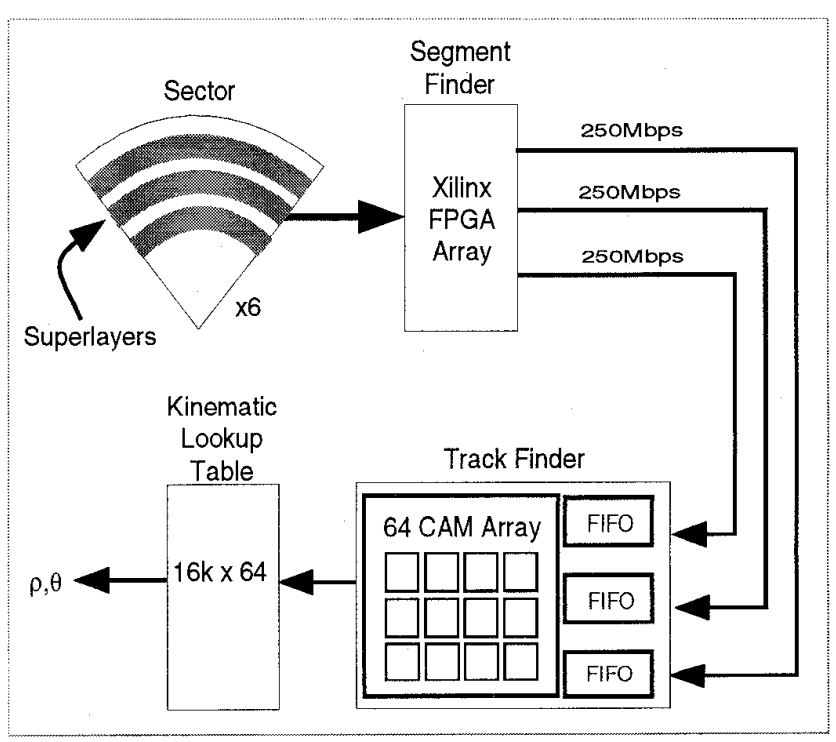

Figure 1. CLAS Level 2 Triggering System 


\section{CAM OPERATION}

The CAM chip currently under development has several features which make it ideal for track finding applications. One such feature is that the CAM is capable of independent byte matching within each 4 byte word. When the CAM is in Compare mode, incoming data are compared with the data in each word of the CAM. Data matches are stored for each byte independently. When the number of matched bytes per word exceeds a threshold, the entire word is said to have matched. This characteristic allows time independent byte streams to be linked together during the compare process.

Figure 2 illustrates the independent byte matching feature of the CAM. Note that only three columns are shown since that is all that CEBAF's track finding algorithm requires, although the CAM supports four byte-wide columns. In Figure 2a, the CAM has been loaded with match data and the Track Finder's input FIFOs have data, but there are no byte or word matches. In Figure $2 b$, the first data set is compared to the CAM data resulting in two byte matches in the right column. In Figure $2 \mathrm{c}$ the second data set is compared resulting in seven additional byte matches, resulting in two matched words. In this example the byte threshold to determine a word match would be set to three. In general the CAM is capable of matching words with 1 or more, 2 or more, 3 or more, or 4 byte matches. This programmable threshold is controlled by input mode bits and allows an experimenter to set the sensitivity of the CAM matching function.

Following the associative table lookup of the CAM's Compare operation, the Address Prime and Address Report modes allow the addresses of the matched words to be output. The Output Ready signal indicates the presence of matched words, and is used to control the Address Reporting. After the Compare, Address Prime and Address Report operations complete, the CAM's match bits can be reset by the Clear Hit mode. The cycle can then be repeated with new segment data from the next event. A summary of the CAM's operational modes are as follows:

NOP -

Write -

Read -

Compare -

Address Prime -

Address Report -

Clear Valid -

Clear Hit - no operation mode

for initial loading of CAM data for reading back loaded CAM data for comparing incoming data initialize the chip for report mode reporting the matching addresses for invalidating the data for clearing the byte matches

\section{ㅁ Byte Match $\mathrm{XX}$ Byte Value \\ Word Match $\longleftarrow$ Match Compare Data}

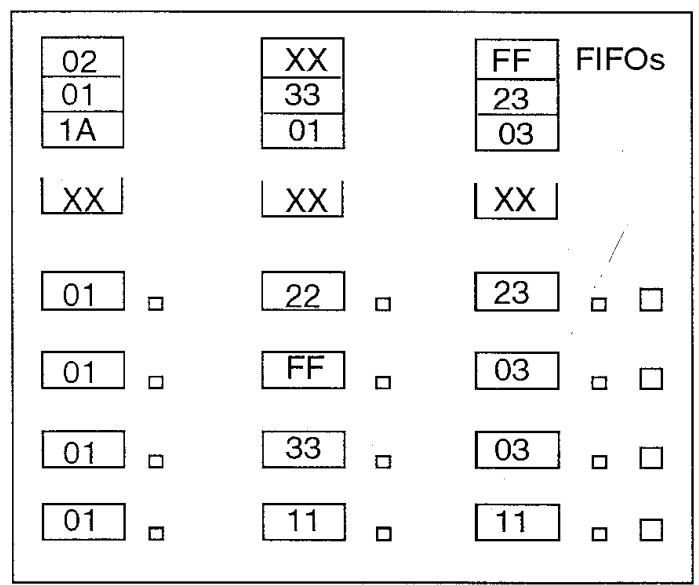

(a)

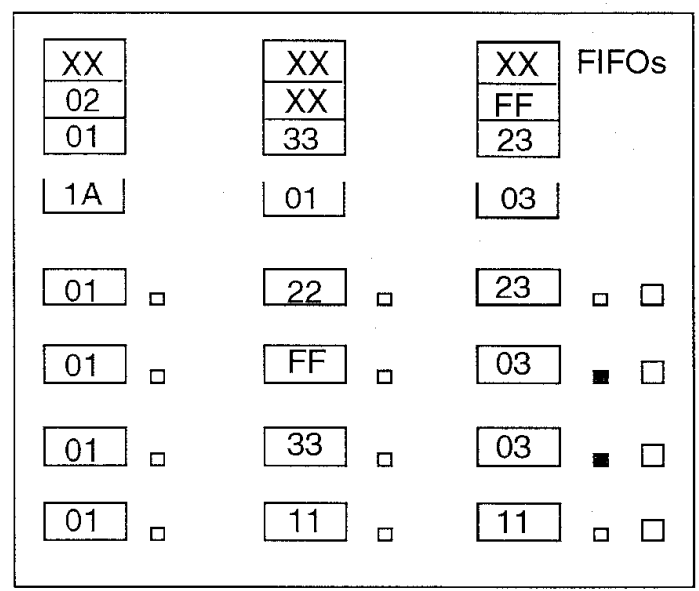

(b)

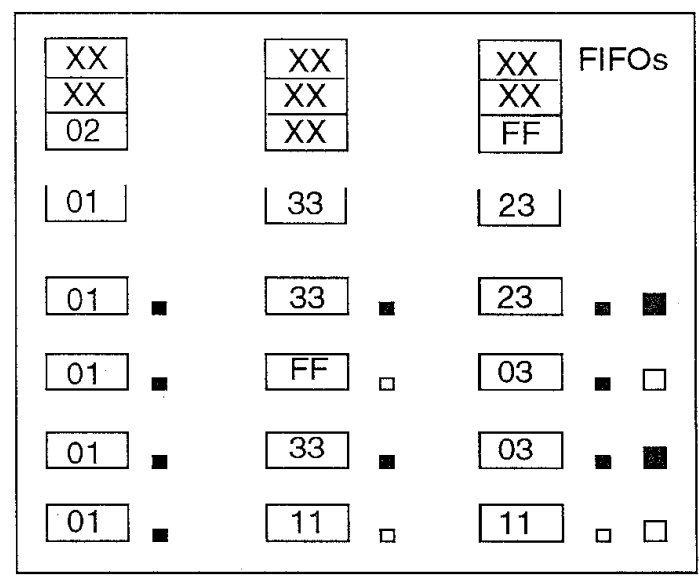

(c)

Figure 2. CAM Byte Matching Example [3] 


\section{CAM HIGH LEVEL ARCHITECTURE}

The high level CAM architecture is shown in Figure 3. The four major functional units are the Address Decoder, the CAM Cell Array, the Priority Encoder and the Control.

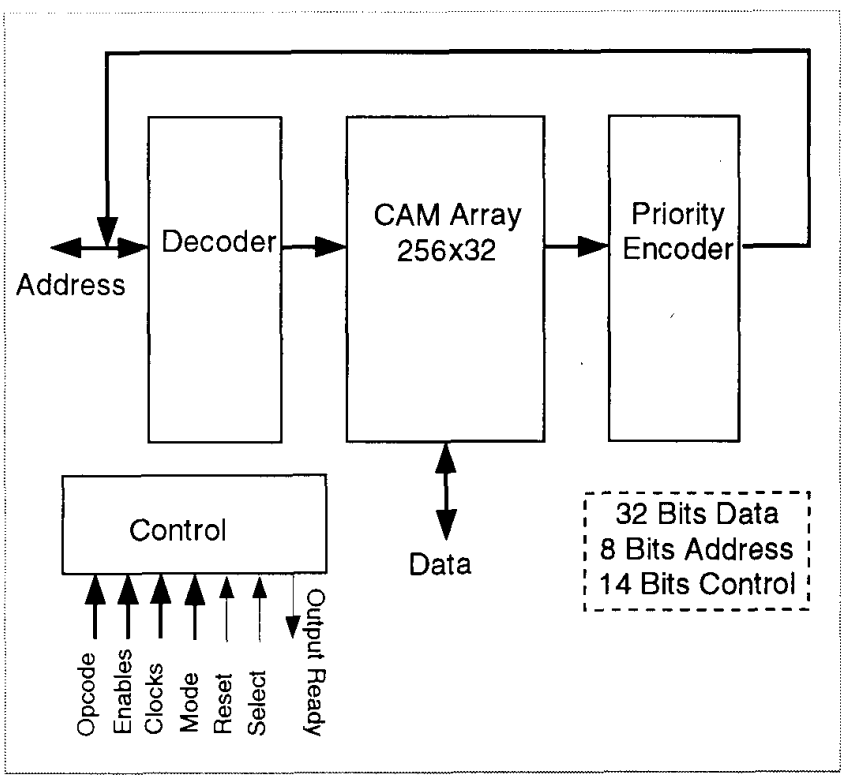

Figure 3. CAM Chip Architecture

The Address Decoder is a straightforward decoder designed using standard cells. It performs an 8 to 256 decode function. It is used during Read, Write and Address Report operations. In Address Reporting the address from the priority encoder is fed back and decoded to invalidate word matches after their corresponding address have been output.

The CAM Cell Array is a 256 word by 32 bit custom memory array. It contains the necessary logic for memory Read, Write and Compare operations. The details of the CAM Cell Array will be discussed further in the next section.

The Priority Encoder is used to form the addresses of the words that match as a result of the Compare operations. These addresses are output during Address Report operations and, as previously stated, are used to invalidate matched words.

The Control block generates timing signals to control data transfers throughout the CAM . All timing is generated relative to two non-overlapping clocks. The operation of the CAM is determined by a 3-bit opcode. Mode bits are used to set the threshold for the CAM's word matching function. The CAM is byte addressable and therefore has four byte enables. A chip select is provided for tri-state output control which is useful when cascading multiple CAMs. An external reset is provided to initialize the CAM's internal control registers to a known state, and the Output Ready is provided to indicate the presence of matched data words within the CAM.

\section{CAM CELL ARRAY ORGANIZATION}

The CAM Cell Array is a $256 \times 32$ array designed with independent byte matching and threshold logic for partial word matching. Most of the CAM Cell Array is custom logic with some standard cells used for the threshold function. The basic Read, Write and Compare operations are standard CAM functions implemented by a nine transistor bit cell. The precharge circuitry, sense amplifiers and data I/O latches are also custom designs.

A functional diagram of the byte design is depicted in Figure 4 . The addition of a Byte Match Latch to a conventional CAM cell is the basis for independent byte matching. Since each byte in the CAM array can record a matched or unmatched status during each compare cycle, multiple data compare cycles may contribute to a word match. The Byte Match Latch is normally reset during an Address Report operation.

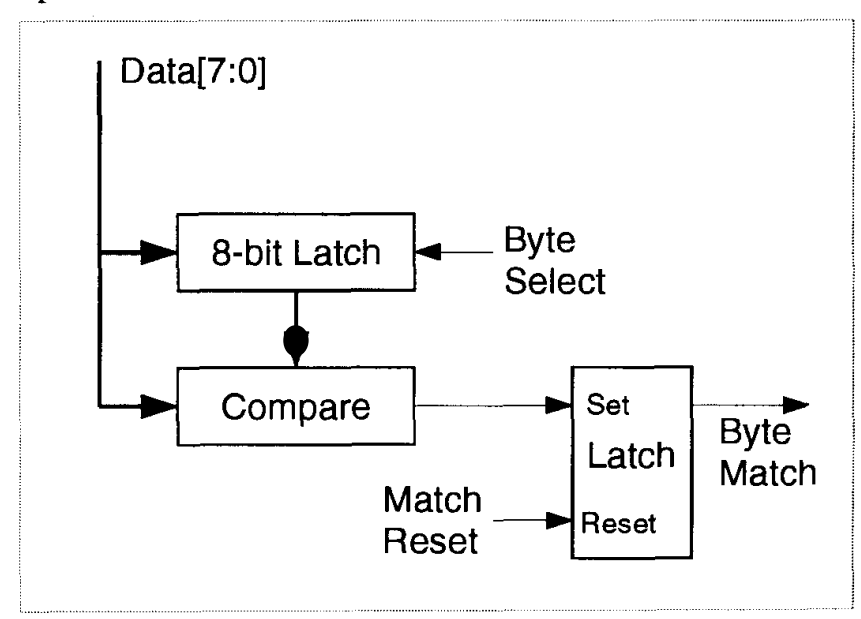

Figure 4. Byte Functional Diagram

Each CAM word consists of four CAM bytes with Threshold Logic and a Valid Bit latch, as shown in Figure 5. During Compare operations, byte match signals are sent to the Threshold Logic. The Threshold logic determines if there is a word match based on the byte matches and the Mode bits. The Mode bits are defined as follows:

Mode Threshold Function

001 or more byte matches implies a word match

012 or more byte matches implies a word match

103 or more byte matches implies a word match

114 byte matches implies a word match

The ability to set the byte match threshold allows the CAM to provide fuzzy matches. This feature may be useful if a 
superlayer occasionally misses a segment.

Each word in the CAM also contains a Valid latch. This is to invalidate CAM words without match data. If a CAM array is only partially filled with valid match data, those words with invalid data will be excluded from the match process. If the threshold logic detects a word match and the word is valid, a Word Match signal is sent to the Priority Encoder to generate its address for output.

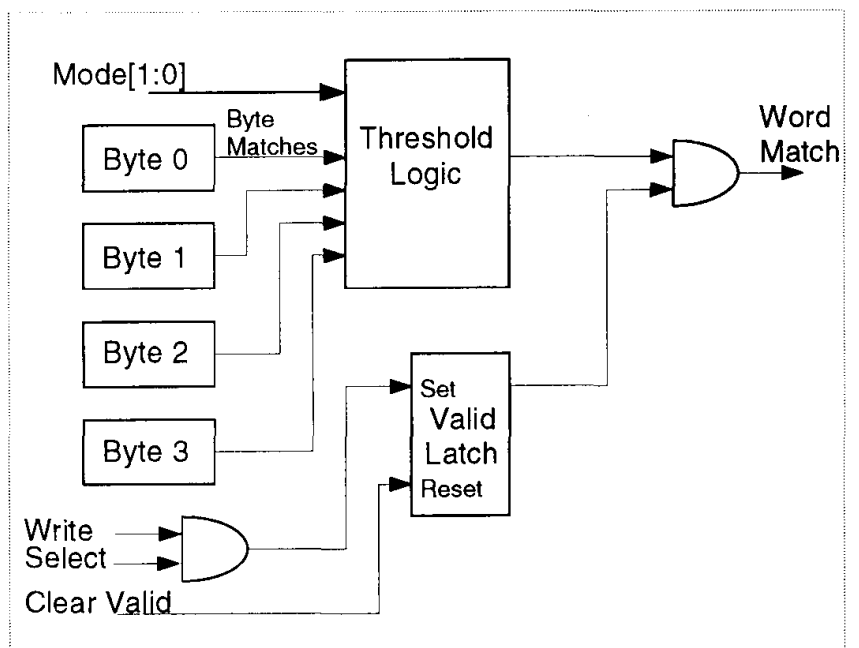

Figure 5. CAM Word Organization

During a Compare operation all data words compare simultaneously and set their corresponding byte match bits. Since the priority encoder can only generate one address at a time, addresses are output sequentially during Address Report operations. Each time a matched word address is reported, its corresponding byte match bits are cleared so the next match address can be encoded for output.

\section{TIMING}

The basic timing of the CAM is shown in Figure 6. Two non-overlapping clocks with a $25 \%$ duty cycle are used. The $\mathrm{CAM}$ is designed to operate at a minimum clock frequency of $12.5 \mathrm{MHz}$. All I/O signals are relative to $\mathrm{Ph} 1$. The $\mathrm{Ph} 2$ clock is used internally to latch the result of Compare operations.

The first group of signals in Figure 6 shows successive write operations. The Write opcode, address and data must be provided. All of these signals are internally latched for the Write operation. Internally the write completes prior to the next Ph1 cycle. In CEBAF's track finder design, conservative timing is used by generating all CAM control signals off of $\mathrm{Ph} 2$. This allows ample time for CAM setup and hold times relative to $\mathrm{Ph} 1$.

The next group of signals shows two consecutive read operations followed by a NOP (No Operation). A Read op-

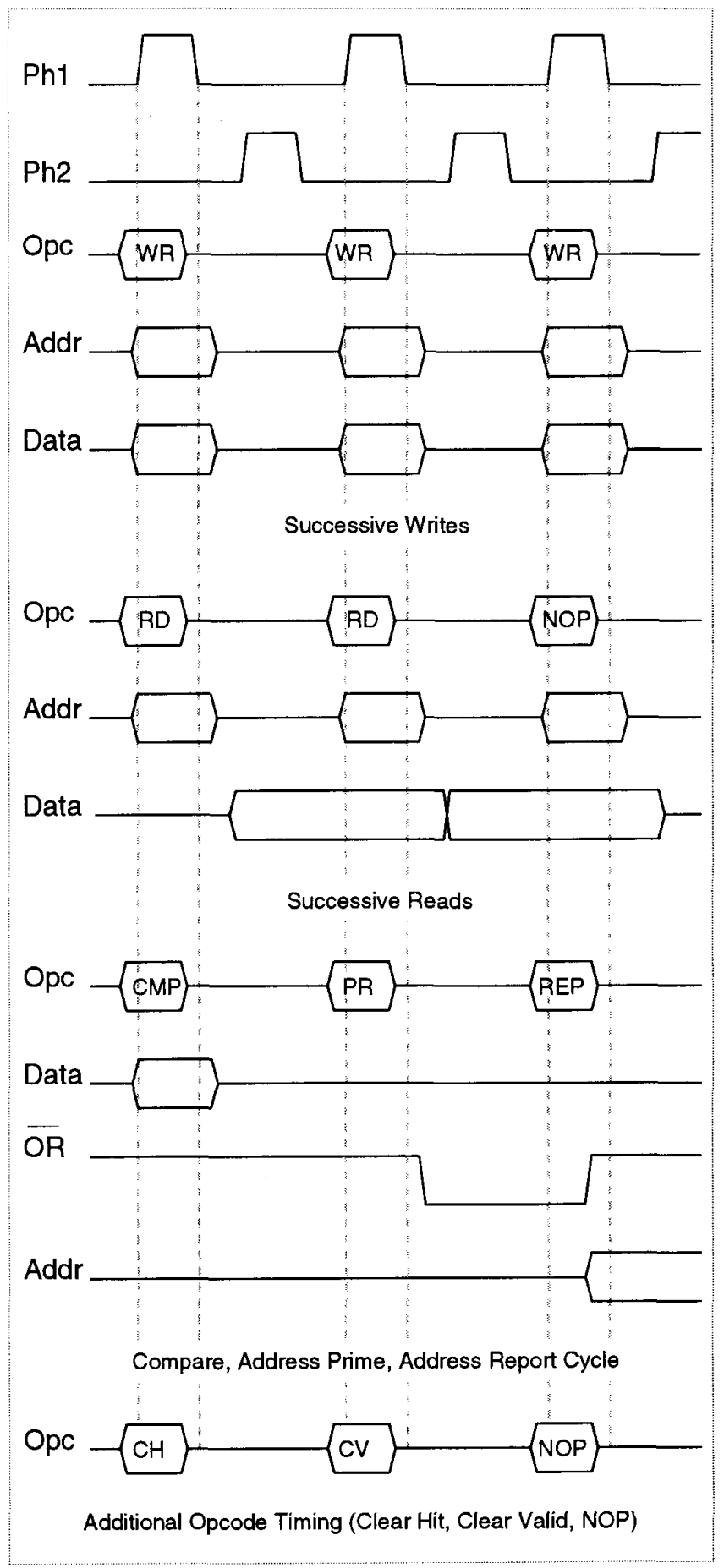

Figure 6. CAM Timing

code and address are provided relative to Ph1. Data will become available after the memory access time and will be held stable through the next Ph1 cycle.

The basic timing for the Compare, Address Prime, Address Report cycle is shown in the third group of signals in Figure 6. The timing shown reflects a single Compare oper- 
ation in which there is a word match. The word match is signified by Output Ready (OR) going low. It is also possible to do successive Compare operations (not shown). This would typically be how the CAM would be used in a track finding application. A single Address Prime opcode is issued to prime an internal address pipeline for the Address Report operation. In the example shown, there was only one match in the CAM and therefore Output Ready goes high signifying no more matches one cycle after the Address Prime. If there were multiple word matches in the CAM, Output Ready would remain low until the last match address is reported by successive Address Report operations. Output Ready goes high on Ph1 of the last match cycle. This is useful in an array of CAM chips as used in the CEBAF triggering system because the array controller can start a Report operation on the next CAM chip without losing any clock cycles.

The last signal in Figure 6 shows the timing of other opcodes. The Clear Hit operation clears all the byte match bits in the CAM. This can be used to reset match bits prior to a new Compare operation. The Clear Valid operation resets all the word valid bits in the CAM. This is useful if new data is to be written into the CAM.

\section{PROTOTYPE DEVELOPMENT}

Thus far four CAM prototype chips have been developed. All prototypes are smaller than the design goal of $256 \times 32$ to reduce development cost. The first three prototypes were designed using a $1.2 \mathrm{u}$ CMOS two layer metal process while the latest prototype scales the design for a $0.8 \mathrm{u}$ CMOS three layer metal process. This prototype is a fully functioning $16 \times 32$ CAM. It is based on the custom byte cell discussed in this paper and uses CMOS standard cells for control and peripheral logic. These prototype samples have been fabricated by MOSIS.

Testing of prototypes has verified the basic development process, custom cell designs, and timing. A sample of the timing captured by a Tektronix DAS $9206 \mathrm{XP}$ from the third prototype is given in Figures 7 and 8. This chip ran successfully at $25 \mathrm{Mhz}$, double the required frequency for the CLAS trigger system.

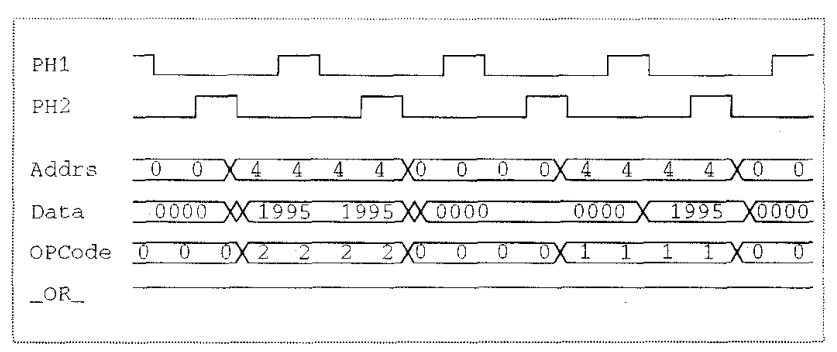

Figure 7. Actual Write/Read timing from CAM prototype operating at $25 \mathrm{MHz}$

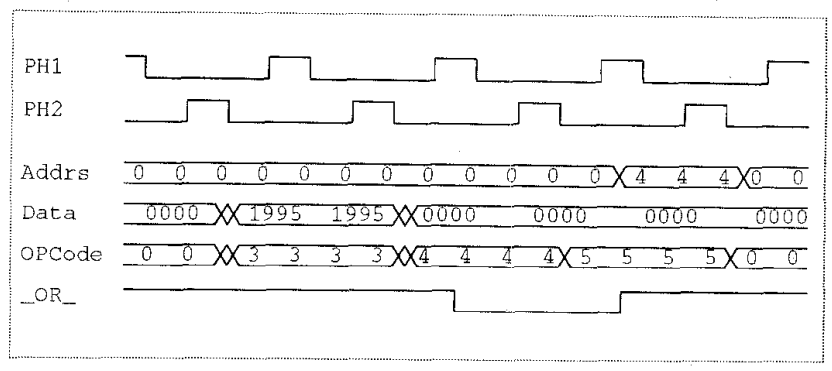

Figure 8. Actual Compare/Prime/Report timing from CAM prototype operating at $25 \mathrm{MHz}$

\section{CONCLUSION}

A 12.5 MHz CMOS 256x32 Content Addressable Memory for fast tracking applications in under development at Christopher Newport University. Basic custom cell designs have been designed and fabricated. A 16×32 fully functional CAM has recently been fabricated through MOSIS and is currently undergoing testing. The designs of both the $64 \times 32$ and $256 \times 32$ versions of the CAM are near completion. Testing of prototypes and design simulations have given promising results and we anticipate having 64 word CAMs in the second quarter of 1996 and the first 256 word chips in the fourth quarter of 1996.

\section{ACKNOWLEDGMENTS}

Special thanks to Mark Lantz of the National Security Agency and Thomas A. Shull of NASA Langley Research Center for their support on this project. We also greatly appreciate grant contributions made by CEBAF and NASA used for funding faculty and student researchers.

\section{REFERENCES}

[1] M. Dell'orso and L. Ristori, VLSI Structures for Track Finding, Nuclear Instruments and Methods in Physics Research, Elsevier Science Publishers, North Holland, October 1989.

[2] M. Dell'orso and L. Ristori, A Highly; Parallel Algorithm for Track Finding, Nuclear Instruments and Methods in Physics Research, Elsevier Science Publishers, North Holland, July 1989.

[3] M.H. Bickley, Design of the Level-2 Track Finder Board for the CEBAF Large Acceptance Spectometer, Masters Thesis, Christopher Newport University, Newport News, VA, 1995.

[4] D.C. Doughty Jr., R.F. Hodson, et al, The Use of Content Addressable Memories in the Level 2 Trigger for the CLAS Dector at CEBAF, RT-95, 1995, to be published. 